\title{
Mediación familiar y violencia de pareja*
}

\author{
Deyanira Salazar Villarroel** \\ Eugenia Vinet Reichhardt***
}

\begin{abstract}
RESUMEN
Este estudio analizó las características de la violencia en parejas que asistieron a mediación familiar. A los participantes se les administró una entrevista semiestructurada y una selección de items de S.A.R.A, para indagar la presencia de violencia de pareja. Los datos recogidos fueron analizados en términos estadísticos a través de frecuencias, porcentajes y aplicación de pruebas de asociación entre variables. Los resultados revelan que en la mayoría de los casos de mediación familiar existen o han existido antecedentes de violencia de pareja, lo que instala la necesidad de desarrollar miradas integradoras del fenómeno de violencia de pareja para diseñar herramientas que aborden esta problemática en el ámbito de la mediación familiar.
\end{abstract}

Conflicto - mediación familiar - violencia de pareja

Family mediation and partner violence

\begin{abstract}
This study analyzed the characteristics of violence in couples that have attended to family mediation. Participants were given a semi-structured interview and a selection of items of S.A.R.A., to investigate the presence of partner violence. The data collected were analyzed statistically through frequencies, percentages and implementation of tests of association between variables. The results reveal that in most cases of family mediation, it exists or there has existed a history of partner violence, which installs the need to develop a integrative view of partner violence phenomenon regarding to devise tools that address this issue in the field of family mediation.
\end{abstract}

$$
\text { Conflict - family mediation - partner violence }
$$

* Este estudio presenta resultados de la tesis para optar al grado de Magíster en Psicología Jurídica y Forense, del Departamento de Psicología de la Universidad de La Frontera, realizada por la primera autora bajo la dirección de la segunda autora.

** Psicóloga, Magíster en Psicología Jurídica y Forense, Centro de Mediación CREA, Escuela de Derecho, Universidad Católica de Temuco, Temuco, Chile. dsalazar@uct.cl

*** Psicóloga, Doctora en Psicología Clínica y de la Salud, Profesora de la Universidad de La Frontera, Temuco, Chile.

Artículo recibido el 30 de marzo de 2011 y aceptado para su publicación por el Comité Editorial el 27 de mayo de 2011. 


\section{ANTECEDENTES TEÓRICOS}

$\mathrm{L}$ a Ley $N^{0} 19.968$, que creó los Tribunales de Familia, reconoció a la mediación como una forma legítima de resolución de conflictos para abordar las diversas materias de su competencia. Dentro de los objetivos para la incorporación de la mediación en la jurisdicción de familia, estuvo la búsqueda de un trabajo colaborativo entre las participantes, que hiciera posible el desarrollo de acuerdos creativos y con acento en el futuro, mejorando la comunicación y la relación entre las partes involucradas en el conflicto y posibilitando economía de tiempo, dinero y energías. La incorporación de la mediación buscó ofrecer un espacio consensuado a las partes donde la resolución de sus conflictos se hiciera de forma más eficiente y respetando sus intereses.

La Ley de Tribunales de Familia en su artículo No 106 se refiere a los principios que rigen la mediación, entre los cuales están la igualdad entre las partes, el interés superior del niño, niña o adolescente, la confidencialidad de la información y la voluntariedad para participar el proceso. En ese contexto, en julio de 2006 se presenta un proyecto de reforma a la Ley de Tribunales de Familia en la que entre otros aspectos repone la figura de la mediación obligatoria contenida en el proyecto de ley original. El texto aprobado establece la derivación obligatoria a mediación, previa a la interposición de la demanda, en todos aquellos asuntos que versen sobre el derecho de alimentos, cuidado y crianza personal y régimen comunicacional de los padres con sus hijos. En estos casos, la mediación es gratuita para todos los usuarios y el Estado cubre los costos de los servicios a través de un sistema de mediadores licitados. En síntesis, el establecimiento de mecanismos alternativos de resolución de conflictos en el sistema de justicia de familia respondió a diversos objetivos de políticas públicas. Por una parte, mejorar el acceso al sistema judicial, ofrecer respuestas de acuerdo más adecuadas a la especial naturaleza de los conflictos de familia y finalmente mejorar la gestión de los tribunales contribuyendo a su descongestión.

El conflicto entre las parejas es un proceso inevitable que puede adquirir distintas manifestaciones, dentro de las que se encuentra la violencia de pareja. Al respecto, López y Pueyo refieren que "la violencia contra la pareja es cualquier intento, amenaza o violencia real perpetrada por un hombre o una mujer contra alguien con quien se tiene, o ha tenido, una relación sentimental íntima" ${ }^{1}$. En este sentido, la justicia de familia no puede estar ajena a esta problemática y debe ofrecer respuestas satisfactorias dirigidas a preservar las relaciones entre los miembros de la familia; cuando se produce disolución de la pareja, es en este contexto donde adquiere relevancia la mediación familiar.

${ }^{1}$ Pueyo, A.; López, S. y Álvarez, E., "Valoración del riesgo de violencia contra la pareja por medio de la SARA”, en Papeles del Psicólogo, vol. 29, 2008, pp. 107-122. 


\section{PERSPECTIVAS TEÓRICAS SOBRE LA VIOLENCIA DE PAREJA}

De acuerdo a Paz Haz "la violencia conyugal hace referencia a toda situación de abuso que se produce entre los miembros de la pareja, de manera cíclica y cada vez con mayor intensidad, es un patrón de interacción que lesiona la integridad física, emocional y sexual de las personas que conforman la pareja" ${ }^{2}$. Esta forma de violencia se puede clasificar en tres categorías:

a) Maltrato contra la mujer: se trata de una violencia basada en el género; se entiende como el maltrato (físico, sexual o psicológico) intencional provocado a toda persona del sexo femenino por su esposo o por aquel hombre con quien ésta mantiene una relación o vínculo íntimo.

b) Maltrato contra el hombre: hace referencia a los malos tratos, ya sean físicos, psicológicos o sexuales, que padece el hombre en el ámbito de la relación íntima que mantiene con una mujer.

c) Violencia cruzada o recíproca: se refiere a aquella situación en que los miembros de la pareja se atacan física, sexual o psicológicamente de manera recíproca.

Los desarrollos teóricos sobre violencia de pareja han ido desde el análisis de la violencia desde la perspectiva de género hasta la exploración del fenómeno desde una mirada sistémica, centrándose en la existencia de pautas relacionales entre los miembros de la pareja.

El autor Calquín plantea que "la violencia desde una perspectiva de género supone conceptualizar que las relaciones en nuestra sociedad no sólo se basan en una distribución desigual del poder (relaciones definidas como asimétricas), sino que son parte constitutiva de la construcción social, de la subjetividad femenina y masculina. Esta distinción permite que los hombres aparezcan estadísticamente como principalmente agresores y las mujeres, como principalmente víctimas. Desde la mirada de género, la violencia en la familia devela un fenómeno estructural inherente a la hegemonía patriarcal, que debe analizarse en relación directa con las estructuras sociales y representaciones colectivas que continuamente son producidas y reproducidas como normales" 3 . Por su parte, Curi y Gianella plantean que "el género es el concepto que organiza a hombres y mujeres y acerca del cual cada cultura sostiene una construcción determinada. La cultura indica qué es ser hombre y mujer, qué actitudes, roles y expectativas en general son esperables en las personas, según su sexo biológico. El sistema patriarcal de género, aún imperante, sostiene un ordenamiento de la distinción hombre-mujer; dicotomiza las diferencias, las

${ }^{2}$ Haz, P., Violencia intrafamiliar, Presidencia de la República, Colombia, 2000, pp. 73-78.

${ }^{3}$ Calquín, C., "Perfil psicosocial de la población usuaria del programa de atención y prevención de violencia intrafamiliar durante el año 2006 en Lo Espejo, Región Metropolitana de Santiago”, Chile, en Revista Terapia Psicológica, 2007, vol. 25, pp. 85-93. 
jerarquiza en una relación de dominación-subordinación y legitima esta jerarquización apoyándose en una naturalización de las características de cada sexo" 4 .

Desde una conceptualización integradora, otros autores han analizado la violencia de pareja desde la perspectiva de la existencia de pautas relacionales que son generadas por ambos miembros de la pareja. Al respecto, Arredondo, Millán y Lira refieren que "el estudio de la violencia se ha abordado desde una perspectiva individual, donde una parte importante de la literatura se ha focalizado en establecer correlaciones entre las características de personalidad del agresor y la perpetración de la violencia. Ante este tipo de estudios se hace necesario considerar variables o factores de carácter relacional para profundizar el marco explicativo existente" .

En este sentido, Perrone y Nannini plantean un modelo teórico caracterizado por un enfoque interaccional, describiendo los circuitos comunicacionales que sostienen la violencia. Esta perspectiva no busca los motivos de la existencia de la violencia, sino que describe un juego relacional particular que la hace posible y que la sostiene en la historia de las relaciones familiares. En el enfoque interaccional, el foco de observación está dirigido a los aspectos comunicacionales del fenómeno de la violencia familiar, concibiéndolo como una secuencia de transacciones en la que todos los participantes son actores responsables en la interacción. Estas transacciones, en la repetición, se establecen como una pauta o regla de relación en el sistema ${ }^{6}$.

Las relaciones que incluyen la violencia no escapan a esta forma de organización de acuerdo a reglas que se instauran en la historia de las interacciones. Se señala que las relaciones familiares violentas muestran determinadas pautas organizadas de interacción que pueden ser categorizadas en tres formas básicas y una variante de una de ellas:

a) Violencia agresión: es una forma de relación violenta que se construye sobre una pauta simétrica, es decir, una pauta de relación en la que A y B se encuentran en una actitud de igualdad y de competencia. Se produce una agresión mutua y bidireccional que se manifiesta a través del intercambio de golpes, insultos, gestos o actitudes violentas recíprocas. La identidad y autoestima de cada actor está preservada, el otro es reconocido como otro y el enfrentamiento se define desde el rechazo y no desde la desconfirmación. Los episodios violentos son disonantes y provocan malestar para los actores.

b) Violencia castigo: se construye sobre una pauta complementaria, es decir, una relación en la que ambos actores han acordado una diferencia entre ellos y una relación de mutua adaptación. Ambos aceptan que no tienen un mismo estatus en

${ }^{4}$ Curi, S., "Mediación y violencia familiar en el contexto judicial", en Revista La Ley, vol. 3, 2002, pp. 305-315.

${ }^{5}$ Arredondo, V., Millán, R. y Lira, G., "Violencia Intrafamiliar: Un estudio exploratorio de factores sistémicos”, en Anales V Congreso Iberoamericano de Psicología Jurídica, Servicios Gráficos, Santiago de Chile, 2003, pp. 493-504.

${ }^{6}$ Perrone, R. y Nannini, M., Violencia y abusos sexuales en la familia, Paidós, Buenos Aires, 2005, pp. 33-48. 
la relación y que, mientras uno propone, el otro acepta. Se produce violencia unidireccional e íntima; el actor en posición alta es quien ejerce la violencia y ambos sostienen estrategias de ocultamiento hacia el entorno social. Se observa una marcada diferencia de poder entre uno y otro. La identidad del actor en posición baja está severamente afectada, en tanto se niega el derecho a ser otro; la relación no se basa en el rechazo sino en la desconfirmación del actor en posición baja. El actor en posición alta manifiesta una mínima conciencia de la violencia y un confuso sentimiento de culpabilidad.

c) Violencia episódica o reactiva: se caracteriza por la ausencia de una pauta estable de relación violenta, se presentan episodios de violencia ligadas a crisis: ruptura de pareja, problemas laborales, problemas económicos, entre otros. Las partes refieren una preocupación por el daño que se puede haber causado a la familia, lo que se acompaña con el deseo de reparación afectiva.

En relación a las manifestaciones de la violencia, se entiende por violencia física aquellos actos que tienen intención de causar daño físico a otro; en tanto que la violencia psicológica se refiere a aquellos actos verbales (o no verbales) que, simbólicamente, dañan a otro o amenazan con causarle daño. La violencia sexual se refiere a las críticas y/o burlas a las que se ve enfrentada una persona respecto a su sexualidad, como así también a la obligación a distintas formas de acercamiento sexual en contra de su voluntad, mediante la utilización de objetos o armas que puedan producirle lesiones de variada intensidad.

Por su parte, Pueyo, López y Álvarez plantean que "la violencia contra la pareja es un conjunto complejo de distintos tipos de comportamientos violentos, actitudes, sentimientos, prácticas, vivencias y estilos de relación entre miembros de una pareja íntima que produce daños, malestar y pérdidas personales graves a la víctima. La violencia contra la pareja no es sólo un sinónimo de agresión física, es un patrón de conductas violentas y coercitivas que incluye los actos de violencia física contra la pareja pero también el maltrato y abuso psicológico, las agresiones sexuales, el aislamiento y control social, el acoso sistemático y amenazante, la intimidación, la humillación, la extorsión económica y las amenazas más diversas". En relación a la presencia de indicadores de violencia de pareja, estos autores señalan que se trata de características asociadas con un incremento de la probabilidad de que sucedan hechos de violencia; en tanto que la presencia de factores protectores reduce la probabilidad de aparición del suceso.

En la violencia contra la pareja se describen una diversidad de indicadores que tienen un papel de mayor o menor intensidad en el inicio, mantenimiento y agravamiento de las distintas formas de violencia ${ }^{7}$. De acuerdo a Pueyo y Redondo, entre estos indicadores se distinguen el ser testigo o víctima de violencia en la niñez o adolescencia, el consumo

${ }^{7}$ Pueyo, A., López, S. y Álvarez, E., "Valoración del riesgo de violencia contra la pareja por medio de la S.A.R.A.”, en Papeles del Psicólogo, vol. 29, 2008, pp. 107-122. 
de alcohol y la violencia hacia otros miembros de la familia en la niñez, el desempleo, bajo nivel de ingresos, bajo nivel educativo y falta de asertividad ${ }^{8}$.

En Chile la preocupación gubernamental por el tema de la violencia intrafamiliar surgió en la década de los noventa. En 1991 se creó en Servicio Nacional de la Mujer. La promulgación de la Ley de violencia intrafamiliar en 1994 se constituye en un hecho importante que tipifica la violencia como un hecho punible. Las investigaciones acerca de violencia de pareja se han centrado en dimensionar la prevalencia de esta problemática. En nuestro país los primeros estudios en torno a la violencia conyugal surgieron en los años 80 a partir de las organizaciones no gubernamentales. A pesar de los esfuerzos realizados en la última década frente a este tema, existe una carencia significativa de investigaciones a nivel nacional, existiendo sólo un estudio de prevalencia en una muestra poblacional realizada por Larraín (1994) con 1.000 mujeres de la Región Metropolitana. Los resultados indicaron que 25,9\% de las encuestadas declaró haber vivido violencia física y 33,9\% reconocieron violencia psicológica. Sólo $40 \%$ de la muestra no presentaba ningún tipo de violencia?

Más adelante, en el año 2001, se realizó un segundo estudio de prevalencia en el que, a diferencia del realizado en 1992, se amplía la muestra de la Región Metropolitana a la Región de La Araucanía. En esta investigación, son más las mujeres que reconocen haber vivido violencia física por parte de su pareja que en el año 1992. En ambas regiones, aproximadamente el $50 \%$ de las mujeres ha vivido violencia en su relación de pareja. Entre los factores asociados a la violencia, en las mujeres se encontró que haber sido testigo de violencia entre los padres, el bajo nivel de escolaridad, la carencia de empleo remunerado, el consumo de alcohol y la falta de redes de apoyo vecinal se correlacionan significativamente con la presencia de violencia conyugal.

\section{MEdiACIÓN FAMILIAR Y VIOLENCIA DE PAREJA}

De acuerdo a Bernal, "la mediación es una técnica pacífica de resolver conflictos donde el protagonismo lo tienen las partes, cambiando el rol de los actores intervinientes en la situación conflictiva, responsabilizándose de sus decisiones y abriendo la puerta para que puedan seguir relacionándose en el futuro. La mediación conceptualiza el conflicto desde una óptica positiva de manera que promueve un cambio en la interpretación de la situación que genera alternativas conducentes a salir del conflicto y donde los intereses

${ }^{8}$ Pueyo, A., Redondo, S., "Predicción de la violencia: Entre la peligrosidad y la valoración del riesgo de violencia”, en Papeles del Psicólogo, vol. 28, 2007, pp. 157-173.

${ }^{9}$ Vizcarra, M.; Cortés, J.; Bustos, L.; Alarcón, M. y Muñoz, S., "Violencia conyugal en la ciudad de Temuco. Un estudio de prevalencia y factores asociados", en Revista Médica de Chile, vol. 129 (12), 2001, pp. 1405-1412. 
de ambas partes son tenidos en cuenta a partir de la interpretación comprensiva de la situación" ${ }^{10}$.

Con respecto a las características del proceso de mediación, González-Capitel refiere que para entender el funcionamiento de la mediación es necesario conocer sus elementos:

a) Voluntariedad: se basa en la decisión de las partes para iniciar, participar y continuar con el proceso de mediación. En cualquier momento las partes tienen el derecho a retirarse, sin la obligación de continuar.

b) Confidencialidad: antes de comenzar la mediación, el mediador entrega a la partes un convenio de confidencialidad que todos suscriben y en el que todos se obligan a no difundir públicamente lo que allí se trate.

c) Imparcialidad o neutralidad: el mediador no toma partido ni se inclina por ninguna de las partes, él debe administrar y controlar el procedimiento, dirigirlo, marcar las pautas y facilitar las alternativas.

d) Flexibilidad: está ligada a la informalidad del procedimiento y hace que sea un sistema más rápido que el sistema legal. Se realizan sesiones conjuntas entre las partes y el mediador y también sesiones privadas entre el mediador y cada una de las partes ${ }^{11}$.

El procedimiento en la mediación posee una estructura básica dividida en cinco etapas:

1) Sesión conjunta inicial: se realiza el discurso de apertura, la explicación de los objetivos y las características del proceso y presentación de las partes y del mediador, quien debe mantener el equilibrio entre las partes.

2) Relato de antecedentes y posiciones de las partes: el mediador da la palabra a cada una de las partes a fin de que éstas hagan una breve historia del conflicto y fijen sus respectivas posiciones.

3) Generación de opciones: el mediador debe diferenciar las posiciones de las partes, de sus intereses y necesidades, intentando captar las emociones, sentimientos y valores.

4) Búsqueda de la solución: en esta etapa se busca un diálogo directo entre las partes para generar una actitud cooperativa en la búsqueda de solución al conflicto.

5) Solución definitiva al problema: en esta fase se detectan los puntos de coincidencia de las partes, luego de lo cual el mediador coordina estas necesidades, de modo que puedan satisfacerse las de ambas partes. Finalmente, se redacta un acuerdo, a fin de

\footnotetext{
${ }^{10}$ Bernal, T., "La mediación en ruptura de pareja", en Revista Centro de Resolución Alternativa de Conflictos, vol. 4, 2003, pp. 67-88.

${ }^{11}$ González-Capitel, C., Manual de Mediación, Atelier, Barcelona, 1999, pp. 17-29.
} 
que en forma clara y precisa se establezcan los distintos puntos de la transacción e incluso las consecuencias para el caso de incumplimiento de una de las partes.

La mediación aplicada al tema familiar es una forma de abordar la separación o el divorcio, enseña a las parejas a separarse y al mismo tiempo a mantener su responsabilidad como padres, posibilitando que los hijos mantengan una relación adecuada después de la separación. El procedimiento de mediación ayuda a toda la familia a realizar la ruptura de la manera menos traumática, estimulando en las partes que decidan cómo quieren regular su vida futura, teniendo en cuenta al otro y los intereses del niño. El propósito de la mediación no es cambiar la decisión de separarse o divorciarse, sino dar a la pareja la oportunidad de hacerlo en forma menos conflictiva ${ }^{12}$.

En el ámbito de la mediación familiar, se observa que no se han realizado investigaciones relacionadas con la temática de violencia, menos aún que se centren en las características y manifestaciones que puede presentar violencia de pareja; por ende, este tema se constituye en un campo inexplorado y requiere del levantamiento de datos que permitan orientar los procedimientos para el abordaje de casos que presenten antecedentes de violencia.

En los procesos de ruptura de pareja es posible que existan o hayan existido situaciones de violencia. Al respecto, Suares plantea que "no es posible negar o invisibilizar esta realidad. Los mediadores que trabajan en este campo se van a encontrar casi siempre con situaciones con un alto contenido emocional, pero además van a escuchar relatos de episodios de violencia. Una de las características de las mediaciones familiares es que en ellas se "ventilan" temas de violencia"13.

Por su parte, Cárdenas refiere que "la mayoría de las familias que llegan a mediación han pasado por episodios violentos en los períodos inmediatamente anterior y posterior a la separación; si ésta ha cesado, la situación es perfectamente mediable pues se trata de casos "con violencia". Si no, el mediador debe exigir que cese la violencia antes de empezar o proseguir la mediación, y puede aconsejar o acompañar a los familiares para que la situación de no violencia se produzca"14.

Por su parte, Curi y Gianella refieren que "los episodios de violencia ligados a la crisis de la separación pueden ser contenidos por el encuadre de la mediación, como uno de los problemas a conversar durante el proceso. Las diferencias más claras en relación a los casos en que la violencia es una pauta estable aparecen en el reconocimiento de ambas partes de los episodios violentos, en el malestar que han generado en ambos, en la posibilidad de reflexión individual y conjunta sobre estas situaciones y en la preocu-

12 Bernal, T. La mediación. Una solución a los conflictos de ruptura de pareja, Colex, Madrid, 1998, pp. 23-27.

13 Suares, M., Mediando en sistemas familiares, Paidós, Buenos Aires, 2002, pp. 373-387.

${ }^{14}$ Cárdenas, E., La mediación en conflictos familiares, Lumen, Buenos Aires, 1998, pp. 209-235. 
pación sobre el daño que puedan haber causado en cada miembro de la pareja y en sus hijos" 15 .

Los profesionales que se dediquen a la mediación familiar deben poseer conocimientos acerca de las características de la violencia de pareja para poder detectarla y para que su intervención no sea iatrogénica. Además, en muchos casos el concurrir a un centro de mediación puede ser un primer intento de romper con esta dinámica, abriendo la posibilidad de que sean derivados a un centro de atención para abordar su problemática.

En relación al rol del mediador, Ortemberg plantea que "el mediador no puede intervenir en el instante en que está aconteciendo un estallido de violencia. Estas situaciones dan lugar a una intervención de especialistas, cuyo objetivo debe ser la contención de quien padece la crisis, pero luego del estallido nada impide la intervención del mediador y el caso será de mediación en violencia familiar" ${ }^{16}$.

En Chile existe escasa literatura y no se han realizado investigaciones que aborden el tema de la mediación y violencia de pareja; de igual forma se ha observado una exigua difusión de experiencias de mediación en este ámbito, existiendo escasa profundización y avances en relación a las formas de intervención que requieren los casos que presentan antecedentes de violencia.

En este sentido, es posible afirmar que el ámbito de la Mediación y del Derecho de Familia no puede estar ajeno a la problemática de la violencia de pareja, por ende, se hace necesario establecer las bases para realizar intervenciones más especializadas que permitan sacar a la violencia del ámbito privado, enfrentarla y buscar alternativas para extinguirla. El proceso de mediación puede colaborar con el proceso de ruptura de pareja, con el objetivo de negociar el funcionamiento que adquirirá el sistema posterior a la separación. Las partes pueden acordar enfrentar la violencia como un problema compartido y co-construir un proceso colaborativo de afrontamiento, a través del reconocimiento de pautas interaccionales destructivas ${ }^{17}$.

En el procedimiento de mediación en casos con antecedentes de violencia es conveniente, en principio, que ambas partes no estén presentes en el momento en que se realizan las negociaciones propias de la mediación. Pueden no coincidir en el espacio estando en salas separadas. El transcurso de la negociación indicará al mediador la conveniencia o no de que las partes se encuentren, considerando el modo en que se sucedan las conversaciones.

En relación a estos temas es relevante y necesaria la realización de estudios a nivel nacional, que busquen detectar la existencia de violencia de pareja en casos de mediación familiar y que puedan identificar las características con que se manifiesta la violencia en la pareja precisando si se trata de violencia reactiva al proceso de ruptura o si estamos

${ }^{15}$ Curi, S., "Mediación y violencia familiar en el contexto judicial", en Revista La Ley, vol. 3, 2002, pp. 305-315.

${ }^{16}$ Ortemberg, O., Mediación en la violencia familiar y en la crisis de la adolescencia, Editorial Universidad, Buenos Aires, 2002, pp. 59-82.

${ }^{17}$ Cid, A., Momberg, M. y Salazar, D., "Mediación y VIF: de la negación a la intervención”, en Revista Centro de Resolución Alternativa de Conflictos, vol. 5, 2005, pp. 125-132. 
en presencia de una pauta interaccional de violencia histórica e instituida entre los miembros de la pareja como estrategia de resolución de conflictos.

\section{OBjetivos}

El objetivo general de esta investigación es identificar y describir las características que presenta la violencia de pareja en casos que asisten a mediación familiar.

Los objetivos específicos son:

a) Identificar el número de parejas que asisten a mediación familiar que presentan indicadores de violencia de pareja.

b) Describir las manifestaciones que adquiere la violencia (física, psicológica, verbal, sexual) en las parejas que asisten a mediación familiar.

c) Describir el tipo de violencia (reactiva a crisis o como pauta de interacción) existente entre los miembros de la pareja que asisten a mediación familiar.

d) Identificar las características de la violencia de pareja que aparecen en el grupo de hombres y en el grupo de mujeres.

\section{Metodología}

\section{Participantes}

La población objetivo estuvo constituida por parejas que asistieron a procesos de mediación familiar en la comuna de Temuco. La muestra fue no probabilística y, por conveniencia, reclutada desde el Centro de Resolución Alternativa de Conflictos, CREA, de la Escuela de Derecho de la Universidad Católica de Temuco, en razón a criterios de accesibilidad y conveniencia. El tamaño muestral fue de 50 parejas, con un total de 100 sujetos.

\section{Diseño}

En la investigación se utilizó un diseño no experimental de carácter descriptivo transversal, es decir, se describieron las variables de manera natural, tal como se encontraron en los sujetos en un solo tiempo.

\section{Técnicas de evaluación}

Para la caracterización de la muestra se aplicó una entrevista semiestructurada que permitió indagar la presencia del fenómeno de la violencia y recoger antecedentes históricos de cada uno de los miembros de la pareja, datos de la historia familiar y de las características de la relación de pareja. Este protocolo se organizó en cuatro 
apartados: Información sociodemográfica; Antecedentes históricos de la familia de origen; Experiencias de violencia en la familia de origen; Antecedentes del fenómeno de violencia en la última relación de pareja.

Para el cumplimiento de los objetivos de la presente investigación, fueron seleccionados algunos ítems de la Spouse Assault Risk Assessment (S.A.R.A). Esta es una guía de valoración del riesgo de violencia desarrollada originalmente por Kropp, Hart, Webster y Eaves (1995) y adaptada al español en 2005 por Andrés-Pueyo y López. Esta guía permite valorar el riesgo de violencia grave entre miembros de una pareja en demandas civiles que enfrentan a las parejas o ex parejas, litigios por custodia de los hijos, separación y divorcio, denuncias penales por malos tratos, valoración del riesgo de reincidencia, estimación del riesgo de violencia física inminente, entre otras ${ }^{18}$.

La selección de los ítems de la S.A.R.A. a utilizar se realizó considerando que este estudio no pretende evaluar riesgo de violencia, sino la presencia, en la actualidad, de violencia en la pareja. Además, dado que la muestra evaluada fue extraída desde el ámbito de familia, los sujetos participantes no asistieron a mediación familiar por motivos de violencia de pareja, sino para regular las materias relativas al derecho de familia (alimentos, relación directa y regular con los hijos, cuidado personal, entre otras) y no presentaron antecedentes judiciales de violencia de pareja.

\section{Plan de análisis}

Los datos consignados en ambos instrumentos fueron analizados estadísticamente a través de la distribución de frecuencias y porcentajes. Se efectuaron análisis de asociación entre variables a través de pruebas no paramétricas y finalmente se efectuó una comparación de grupos en función del sexo de los sujetos participantes donde los datos fueron analizados estadísticamente a través de la distribución de frecuencias y porcentajes.

\section{Resultados}

\section{Caracterización sociodemográfica de la muestra total}

La muestra total estuvo constituida por 50 parejas (100 sujetos) que asistieron a procesos de mediación familiar durante el año 2009. Con respecto a su procedencia, 42 parejas (84\%) asistieron a mediación derivados desde instituciones, tales como Tribunal de Familia y Corporación de Asistencia Judicial (CAJ) y sólo 8 parejas (16\%) solicitaron espontáneamente asistir a un proceso de mediación. En relación a las características sociodemográficas, el promedio de edad fue de 34,9 años (rango entre 18 y 54 años, desviación estándar de 7,7). El nivel educacional presentó una media de 11,13 años de

18 Pueyo, A., López, S. y Álvarez, E., "Valoración del riesgo de violencia contra la pareja por medio de la SARA”, en Papeles del Psicólogo, vol. 29, 2008, pp. 107-122. 
educación formal (desviación estándar de 1,33). En la Tabla 1 se presenta detalladamente de cada una de las variables descritas.

TABLA 1

INFORMACIÓN SOCIODEMOGRÁFICA EN MUESTRA COMPLETA $(\mathrm{n}=100)$

\begin{tabular}{llr}
\hline \multicolumn{1}{c}{ Variables } & \multicolumn{1}{c}{ Categorías } & Porcentaje \\
\hline Procedencia de la causa & Institución & 84 \\
& Espontánea & 16 \\
Materia de la causa & Alimentos & 50 \\
& Relación directa y regular & 22 \\
& Aspectos previos al divorcio & 20 \\
Nivel educacional & Cuidado personal & 8 \\
& Básica completa & 11 \\
& Básica incompleta & 11 \\
& Media completa & 39 \\
Estado civil & Media incompleta & 21 \\
& Superior completa & 8 \\
Situación laboral & Superior incompleta & 10 \\
& Casado (a) & 68 \\
& Soltero (a) & 24 \\
& Otros (divorciado, viudo) & 8 \\
& Trabajo estable & 43 \\
& Labores de hogar & 24 \\
& Trabajo esporádico & 22 \\
& Cesante & 10 \\
& Estudiante & 1 \\
\hline
\end{tabular}

Respecto de los antecedentes históricos de la familia de origen, un $34 \%$ de los sujetos participantes experimentó en su infancia la muerte de alguna figura significativa (padre, madre, otros), un 38\% tuvo como experiencia la separación de sus padres y un $42 \%$ sufrió el abandono por parte del padre o de la madre. Con respecto a la existencia de experiencias de violencia y problemas de salud asociados a la violencia en la familia de origen, un $27 \%$ de los sujetos reportó experiencias de maltrato físico, un $29 \%$ maltrato psicológico y un $3 \%$ maltrato sexual.

Caracterización de la muestra de parejas con antecedentes de violencia según la entrevista

Con las 50 parejas que conformaron la muestra total de esta investigación se realizó un análisis considerando a la pareja como unidad de estudio centrado en aquellas parejas 
que presentaban indicadores de violencia. La muestra quedó circunscrita a 42 parejas que habían utilizado la violencia como forma de resolución de conflictos, esto es, el $84 \%$ de la muestra total de parejas. A continuación se presenta una caracterización de esta submuestra.

En cuanto a las manifestaciones que adquiere esta violencia, se observa que las parejas han utilizado preferentemente la violencia psicológica y verbal, caracterizada por la realización de actos verbales y/o amenazas que se efectúan con la intención de dañar al otro.

Centrándonos en la dinámica que puede adquirir la violencia, se observa que la mayoría de las parejas utilizaron la violencia recíproca, una forma de relación violenta donde se produce una agresión mutua y bidireccional que se manifiesta a través del intercambio de golpes, insultos, gestos o actitudes. Con respecto al tipo de pauta de violencia existente entre los miembros de la pareja destaca la presencia de la pauta de violencia simétrica en la mayoría de las parejas caracterizada como un patrón de relación en la que sus miembros se encuentran en una actitud de igualdad y de competencia.

En relación al tipo de violencia imperante en la relación de pareja, los resultados muestran que, mayoritariamente, la violencia se había establecido como una pauta de interacción, es decir, como la estrategia recurrente utilizada para la resolución de conflictos.

Por último, según el tipo de pauta de interacción asociada a la violencia, la mayoría de las parejas manifestaron la existencia de una pauta de interacción de violencia-agresión, que se caracteriza por una forma de relación violenta construida sobre una pauta simétrica, donde ambos miembros de la pareja se encuentran en igualdad de poder, produciéndose agresiones mutuas y bidireccionales. La información detallada sobre esta características se presenta en la Tabla 2.

\section{TABLA 2}

Características de la muestra de parejas con antecedentes de Violencia

\begin{tabular}{lcr}
\hline \multicolumn{1}{c}{ Variable } & $\mathrm{f}$ & $\%$ \\
\hline Manifestaciones de la violencia* & & 88 \\
Violencia psicológica & 37 & 83 \\
Violencia verbal & 35 & 58 \\
Violencia física & 24 & 83 \\
Dinámica de violencia de pareja & & 12 \\
Recíproca & 35 & 5 \\
Hombre a mujer & 5 & \\
Mujer a hombre & 2 & 81 \\
Tipo de pauta de violencia & & 19 \\
Simétrica & 34 & \\
Complementaria & 8 & \\
\hline
\end{tabular}




\begin{tabular}{lcr}
\hline \multicolumn{1}{c}{ Variable } & $\mathrm{f}$ & $\%$ \\
\hline Tipo de violencia en la relación & & 74 \\
Pauta de interacción & 31 & 26 \\
Reactiva a crisis & 11 & 83 \\
Tipo de pauta de interacción de violencia & & 17 \\
Violencia agresión & 35 & 7 \\
Violencia castigo & 7 & \\
\hline
\end{tabular}

* Pueden coexistir las diferentes categorías.

Caracterización de la muestra de parejas con antecedentes de violencia según los items de la S.A.R.A.

Los resultados de la administración de los ítems seleccionados de la prueba S.A.R.A. en las parejas que presentan indicadores de violencia revelan altos niveles de acuerdo entre los miembros de la pareja en la mayoría de los ítems.

En la sección Ajuste Psicosocial se observó acuerdo en las 42 parejas (100\% de acuerdo intrapareja) en los ítems 4 y 5 . En el ítem 6 se observa acuerdo en 15 parejas $(35 \%)$.

En la sección Historial de violencia contra la pareja, en el ítem se observa acuerdo en 41 parejas (97\% de la muestra). En el ítem 17 el acuerdo llega a 38 parejas (90\%). La información detallada aparece en la Tabla 3.

\section{TABLA 3}

Resultados de los ÍTEMS DE LA S.A.R.A. EN MUESTRA DE PAREJAS CON ANTECEDENTES DE VIOLENCIA PAREJA $(\mathrm{n}=42)$

\begin{tabular}{|c|c|c|c|c|c|}
\hline \multirow{2}{*}{ Variables } & \multirow{2}{*}{ Categorías } & \multicolumn{2}{|c|}{$\begin{array}{l}\text { Valores según } \\
\text { categoría }\end{array}$} & \multicolumn{2}{|c|}{$\begin{array}{c}\text { Acuerdo } \\
\text { intrapareja }\end{array}$} \\
\hline & & $\mathrm{f}$ & $\%$ & $\mathrm{f}$ & $\%$ \\
\hline \multicolumn{6}{|l|}{ Ajuste Psicosocial } \\
\hline $\begin{array}{l}\text { 4. Problemas recientes en las } \\
\text { relaciones de pareja }\end{array}$ & $\begin{array}{l}\text { Severa } \\
\text { Moderada } \\
\text { Ausente }\end{array}$ & $\begin{array}{r}18 \\
23 \\
1\end{array}$ & $\begin{array}{r}43 \\
55 \\
2\end{array}$ & 42 & 100 \\
\hline $\begin{array}{l}\text { 5. Problemas recientes de } \\
\text { ocupación/trabajo }\end{array}$ & $\begin{array}{l}\text { Severa } \\
\text { Moderada } \\
\text { Ausente }\end{array}$ & $\begin{array}{r}13 \\
3 \\
26\end{array}$ & $\begin{array}{r}31 \\
7 \\
62\end{array}$ & 42 & 100 \\
\hline
\end{tabular}




\begin{tabular}{|c|c|c|c|c|c|}
\hline \multirow{2}{*}{ Variables } & \multirow{2}{*}{ Categorías } & \multicolumn{2}{|c|}{$\begin{array}{l}\text { Valores según } \\
\text { categoría }\end{array}$} & \multicolumn{2}{|c|}{$\begin{array}{l}\text { Acuerdo } \\
\text { intrapareja }\end{array}$} \\
\hline & & $\mathrm{f}$ & $\%$ & $\mathrm{f}$ & $\%$ \\
\hline \multirow{3}{*}{$\begin{array}{l}\text { 6. Víctima y/o testigo de } \\
\text { violencia familiar en la infancia } \\
\text { y/o adolescencia }\end{array}$} & Severa & 6 & 14 & \multirow[t]{3}{*}{15} & \multirow[t]{3}{*}{35} \\
\hline & Moderada & 1 & 2 & & \\
\hline & Ausente & 8 & 19 & & \\
\hline \multicolumn{6}{|c|}{ Historial de violencia contra la pareja } \\
\hline \multirow[t]{3}{*}{ 11. Violencia física anterior } & Severa & 6 & 14 & \multirow[t]{3}{*}{19} & \multirow[t]{3}{*}{45} \\
\hline & Moderada & 6 & 14 & & \\
\hline & Ausente & 7 & 17 & & \\
\hline \multirow{3}{*}{$\begin{array}{l}\text { 14. Incremento reciente en la } \\
\text { frecuencia o gravedad de las } \\
\text { agresiones }\end{array}$} & Severa & 22 & 52 & \multirow[t]{3}{*}{29} & \multirow[t]{3}{*}{69} \\
\hline & Moderada & 7 & 17 & & \\
\hline & Ausente & - & - & & \\
\hline \multirow{3}{*}{$\begin{array}{l}\text { 16. Minimización extrema o } \\
\text { negación de la violencia contra } \\
\text { la pareja }\end{array}$} & Severa & - & - & \multirow[t]{3}{*}{41} & \multirow[t]{3}{*}{97} \\
\hline & Moderada & 1 & 2 & & \\
\hline & Ausente & 40 & 95 & & \\
\hline \multirow{3}{*}{$\begin{array}{l}\text { 17. Actitudes que apoyan y } \\
\text { consienten la violencia contra la } \\
\text { pareja }\end{array}$} & Severa & - & - & \multirow[t]{3}{*}{38} & \multirow[t]{3}{*}{90} \\
\hline & Moderada & - & - & & \\
\hline & Ausente & 38 & 90 & & \\
\hline
\end{tabular}

Caracterización de la muestra según sexo

Se realizó un análisis comparativo entre el grupo de hombres y el de mujeres. Los resultados obtenidos en las variables relacionadas con información sociodemográfica y los antecedentes de la historia de vida (nivel educacional, situación laboral, experiencias de violencia y problemas asociados a la violencia en familia de origen) no presentan diferencias significativas a excepción de la variable "situación laboral" donde los hombres reportan valores significativamente diferentes a los de las mujeres $\left(X^{2}=41,903\right.$, nivel de significación $=0,000)$.

Con respecto al fenómeno de violencia en la última relación de pareja se comparó a hombres y mujeres en las siguientes variables: utilización de la violencia, manifestaciones de la violencia, dinámica de violencia de pareja, tipo de pauta de violencia en la relación, tipo de violencia en la relación de pareja y tipo de pauta de interacción de violencia; aunque existen leves diferencias entre hombres y mujeres éstas no son significativas. La información se presenta en la Tabla 4. 
TABLA 4

Antecedentes de Violencia de pareja en la última RelaCión de pareja SEGÚN SEXO

\begin{tabular}{|c|c|c|c|c|}
\hline \multirow{2}{*}{ Variables } & \multicolumn{2}{|c|}{ Hombre $(n=50)$} & \multicolumn{2}{|c|}{ Mujer $(\mathrm{n}=50)$} \\
\hline & $\mathrm{f}$ & $\%$ & $\mathrm{f}$ & $\%$ \\
\hline Utilización de la violencia & 41 & 82 & 47 & 94 \\
\hline \multicolumn{5}{|l|}{ Manifestaciones de violencia } \\
\hline Violencia física & 21 & 42 & 24 & 48 \\
\hline Violencia psicológica & 37 & 74 & 42 & 84 \\
\hline Violencia verbal & 36 & 72 & 42 & 84 \\
\hline \multicolumn{5}{|l|}{ Dinámica de violencia } \\
\hline Recíproca & 36 & 72 & 38 & 76 \\
\hline Hombre a mujer & 5 & 10 & 6 & 12 \\
\hline Mujer a hombre & 2 & 4 & 3 & 6 \\
\hline \multicolumn{5}{|l|}{ Tipo de pauta de violencia } \\
\hline Simétrica & 34 & 68 & 38 & 76 \\
\hline Complementaria & 8 & 16 & 9 & 18 \\
\hline \multicolumn{5}{|l|}{ Tipo de violencia } \\
\hline Pauta de interacción & 32 & 64 & 34 & 68 \\
\hline Reactiva a crisis & 12 & 24 & 14 & 28 \\
\hline \multicolumn{5}{|l|}{ Tipo de pauta de interacción } \\
\hline Violencia agresión & 34 & 68 & 38 & 76 \\
\hline Violencia castigo & 8 & 16 & 9 & 18 \\
\hline
\end{tabular}

Resultados de los ítems de la S.A.R.A. según sexo

Los resultados de la administración de los ítems seleccionados de la prueba S.A.R.A. en función del sexo de los sujetos participantes revelan que no existen diferencias significativas entre hombres y mujeres. En la sección Ajuste Psicosocial, en el ítem 4 (problemas recientes en las relaciones de pareja) un $54 \%$ de las mujeres y un $40 \%$ de los hombres se ubicaron en la categoría severa; en el ítem 5 (problemas recientes de ocupación) un $46 \%$ de las mujeres y $34 \%$ de los hombres estuvieron en la categoría severa y en el ítem 6 (víctima y/o testigo de violencia familiar en la infancia y/o adolescencia) un $36 \%$ de las mujeres y una $30 \%$ de los hombres estuvieron en la categoría severa.

Por otra parte, en la sección Historial de violencia contra la pareja, en el ítem 11 (violencia física anterior) el $34 \%$ de las mujeres y el $24 \%$ de los hombres se posicionaron en la categoría severa; en el ítem 14 (incremento reciente en la frecuencia o gravedad de las agresiones) un $60 \%$ de las mujeres y un $54 \%$ de los hombres estuvo en el rango de severa; en el ítem 16 (minimización extrema o negación de la violencia anterior contra la pareja) un $98 \%$ de las mujeres y $96 \%$ de los hombres se ubicaron en la categoría de 
ausente y, finalmente, en el ítem 17 (actitudes que apoyan y consienten la violencia contra la pareja) un $98 \%$ de las mujeres y $94 \%$ de hombres estuvo en la categoría ausente.

\section{Discusión}

Los resultados de esta investigación tienden a confirmar las posturas teóricas vinculadas al análisis de la violencia de pareja desde la perspectiva interaccional y significan importantes aportes para el trabajo en mediación familiar en parejas con antecedentes de violencia.

La mayoría de las parejas que participaron en la investigación asistieron a procesos de mediación derivados desde una institución, mayoritariamente desde el Tribunal de Familia. Esto puede estar relacionado con las modificaciones introducidas a la ley que hace la derivación a mediación obligatoria para los jueces en tres materias: alimentos, relación directa y regular del padre con los hijos y cuidado personal. En la muestra analizada destaca el alto porcentaje de parejas que asiste a regular el tema de alimentos, lo cual muestra que la principal preocupación de las parejas que se encuentran en procesos de separación es la reorganización del presupuesto familiar y la determinación de recursos disponibles para la satisfacción de las necesidades básicas; ésta suele ser una de las áreas de mayor conflicto pos-separación.

Por otro lado, también aparece un alto porcentaje de parejas que asiste a mediación para organizar la relación directa y regular que tendrán los padres con sus hijos. En este aspecto, las parejas, posterior a la ruptura, se encuentran confundidas y con dificultades para separar los roles de hombre y mujer y el de padre y madre. En esto, el proceso de mediación puede servir de base para que la pareja aprenda la diferencia entre los subsistemas conyugal/parental y logren continuar funcionando y fortaleciéndose en sus roles de padres, con el objetivo de que los hijos se resientan lo menos posible y logren un adecuado desarrollo.

En relación a las características sociodemográficas, menos de la mitad de la muestra posee enseñanza media completa; esto está relacionado con baja calificación laboral, antecedente generador de tensión que podría vincularse con la aparición de violencia de pareja. Con respecto a las experiencias de violencia en la familia, un gran número de los sujetos reveló haber sido víctima de violencia física o psicológica en su infancia por parte de miembros de su familia. Las características expuestas (desempleo, bajo nivel de ingresos, bajo nivel educativo y ser víctima de violencia en la niñez y adolescencia) concuerdan con lo expuesto por Pueyo y Redondo como antecedentes que incrementan la probabilidad de que se produzca violencia en la pareja ${ }^{19}$.

Los principales resultados de este estudio permiten afirmar que la gran mayoría de las parejas estudiadas ha utilizado la violencia como estrategia de resolución de conflictos

19 Pueyo, A., López, S. y Álvarez, E., "Valoración del riesgo de violencia contra la pareja por medio de la S.A.R.A.”, en Papeles del Psicólogo, vol. 29, 2008, pp. 107-122. 
en sus diversas manifestaciones; utilizando, mayormente, violencia psicológica, verbal $\mathrm{y}$, en un porcentaje, menor violencia física.

Con respecto a la dinámica de violencia, los resultados de este estudio revelan que la mayoría de las parejas evaluadas reportaron haber experimentado violencia recíproca, donde ambos miembros de la pareja se atacan mutuamente, aunque utilicen distintas formas de violencia. Esto coincide con lo planteado por Paz Haz, quien señala que la violencia de pareja hace referencia a todas las formas de abuso que se producen entre los miembros de la pareja, que lesiona la integridad física, emocional y/o sexual de las personas que conforman la pareja. La violencia conyugal puede presentarse con distintas dinámicas: violencia contra la mujer, violencia contra el hombre y violencia recíproca o cruzada.

Con respecto a este punto, en el ámbito jurídico ha primado, en el análisis de la violencia de pareja, la mirada de género, referida a todo acto de violencia basado en la pertenencia al sexo femenino y en la relación de superioridad del hombre con respecto a la mujer (relaciones asimétricas) como parte de la construcción social y de la identidad femenina y masculina ${ }^{20}$. Esto se ha traducido en que en la mayoría de los casos donde se ha presentado violencia la mujer sea catalogada como víctima y el hombre como victimario.

Sin embargo, a partir del análisis de los resultados de esta investigación se puede afirmar que en el ámbito de la mediación familiar no es recomendable catalogar la violencia de pareja sólo desde la perspectiva de género, pues sería restrictivo, entrampando al hombre y la mujer en roles de víctima y victimario. En este estudio se observa, mayoritariamente, la existencia de violencia recíproca, en la que ambos miembros de la pareja han ejecutado actos de violencia. Estos antecedentes conducen al desarrollo de perspectivas más integradoras, centradas en la existencia de pautas relacionales generadas por el hombre y la mujer y en las que existe responsabilidad compartida en el origen y mantención de los patrones de violencia. Esta nueva mirada implica devolver la responsabilidad de los actos violentos a sus protagonistas, de manera que puedan recuperar el poder para hacer modificaciones en dichas conductas.

La violencia de pareja como una pauta de interacción tiene su origen en la existencia de circuitos comunicacionales que sostienen la violencia; estos circuitos se traducen en una serie de transacciones en las que los participantes son actores responsables en la interacción. Estas interacciones se establecen como pautas o reglas de relación en el sistema. En este sentido, los resultados de este estudio son coherentes con lo expuesto en los antecedentes teóricos: se observa el predominio de la violencia de pareja establecida como pauta de interacción entre los miembros en contraste con una menor cantidad de casos en los que la violencia surge como respuesta ante determinadas crisis. El proceso de ruptura de pareja produce un incremento y agudización en las manifestaciones de la violencia, la cual es mayor cuando existe una pauta de interacción previa.

${ }^{20}$ Calquín. C., "Perfil psicosocial de la población usuaria del programa de atención y prevención de violencia intrafamiliar durante el año 2006 en Lo Espejo, Región Metropolitana de Santiago”, Chile, en Revista Terapia Psicológica, 2007, vol. 25, pp. 85-93. 
Continuando con el análisis de la violencia de pareja, a nivel teórico, y desde una mirada relacional se describe la existencia de ciertos patrones organizados de interacción, uno de los cuales es la violencia-agresión, caracterizada por ser una forma de relación violenta construida sobre una pauta de relación simétrica en la que ambos miembros de la pareja se encuentran en igualdad de condiciones y equilibrio de poder; consecuentemente, las conductas agresivas son mutuas y bidireccionales. A esto se agrega que los episodios de violencia son disonantes y provocan malestar para los actores. En relación a esto, en el presente estudio la mayoría de las parejas que utilizaron la violencia presentaron una pauta de interacción del tipo violencia-agresión con simetría de poder y manifestaciones de violencia recíprocas; asimismo, las parejas reconocieron que el haber utilizado la violencia le producía malestar y disconformidad.

Al comparar los resultados según sexo, sólo se observan diferencias significativas en cuanto a la situación laboral; más de la mitad del grupo de hombres tiene una ocupación estable en contraste con un alto número de mujeres sin ingreso seguro, dedicadas mayoritariamente a trabajos esporádicos y labores de hogar. Este fenómeno se relaciona con la mayor dependencia económica que presentan las mujeres con respecto a los hombres; además, hace comprensible que la solicitud de alimentos sea la materia predominante en los procesos de mediación: son las mujeres las que demandan alimentos para satisfacer las necesidades de su familia con posterioridad a la separación.

Sobre los antecedentes históricos de separación de los padres, llama la atención que tanto en los hombres como en las mujeres se presenten resultados similares. En ambos grupos se presentó la experiencia de ruptura de pareja en los padres; es decir, los participantes, hombres y mujeres, habían vivenciado el proceso de separación de los progenitores desde su rol de hijos. De igual forma se observa similitud entre ambos grupos en las experiencias de maltrato físico y psicológico en la infancia. Tanto los hombres como las mujeres presentaron antecedentes históricos de maltrato en la infancia que pueden estar vinculados o pueden incrementar la probabilidad de que se manifiesten situaciones de violencia de pareja en la adultez tal como lo señalan los antecedentes teóricos.

En la utilización de la violencia en la última relación de pareja se observa que el grupo de mujeres presenta porcentajes más elevados que el de hombres. Las mujeres aparecen utilizando la violencia en todas sus formas (física, psicológica, verbal), reportando incluso conductas de violencia sexual, expresadas en forma de ridiculización y humillación a la pareja por su desempeño sexual; esto es un elemento importante para analizar el rol tradicional de víctima que ha tenido la mujer dentro de la perspectiva de violencia de género.

Con respecto a la dinámica con que se presenta la violencia de pareja se observa similitud entre ambos grupos pues la mayoría de hombres y mujeres reportaron la existencia de violencia recíproca o cruzada. De igual forma, en ambos grupos se observa que el tipo de pauta de violencia es simétrica con pauta de interacción de violencia-agresión. Además, es destacable que tanto hombres como mujeres señalen que la violencia de pareja se relaciona con la existencia de una pauta de interacción y que no emergió sólo como respuesta ante la crisis; ambos grupos reconocen que el proceso de ruptura de pareja fue un factor que contribuyó al incremento de las manifestaciones de violencia arraigadas 
como pauta de interacción en los miembros de la pareja. Esto concuerda con lo expuesto por Perrone y Nannini respecto a la existencia de una pauta interaccional que sostiene la violencia de pareja, una secuencia de transacciones en la que todos los participantes son actores responsables en la interacción. Estas transacciones, en la repetición, se establecen como una pauta o regla de relación en el sistema ${ }^{21}$.

Estos resultados revelan la existencia de consenso entre los grupos de hombres y mujeres en cuanto a la utilización de la violencia de pareja, se observan similitudes con respecto al reporte de las manifestaciones, la dinámica y tipo de pauta de violencia. Por esta razón, la información recogida plantea la necesidad de actualizar la representación de la mujer como sujeto pasivo y receptor de violencia que ha sido característica de la perspectiva de género, por un planteamiento que posiciona al hombre y la mujer en un plano de mayor igualdad y equilibrio de poder. A esto se suma la necesidad de reflexionar sobre la perspectiva bajo la cual se analiza el fenómeno de la violencia de pareja en nuestro país, y específicamente, en la ámbito de la mediación familiar; se plantea el desafío de desarrollar miradas más integradoras que analicen el fenómeno de la violencia con mayor amplitud, erradicando posturas reduccionistas en las que la violencia se manifiesta unidireccionalmente, desde el hombre a la mujer ${ }^{22}$.

Finalmente, y realizando un análisis integrador de la información recogida en la presente investigación, es posible afirmar que en un alto porcentaje de parejas que asisten a mediación familiar existe o han existido antecedentes de violencia de pareja. Esto resulta relevante, pues aun cuando las materias a mediar no estén directamente ligadas a este fenómeno, es factible encontrar hechos de violencia en parejas que asisten a mediación para la regulación de materias como alimentos, relación directa y regular, entre otros.

En esta línea, y de acuerdo a los antecedentes y resultados analizados, queda de manifiesto la posibilidad de realizar procesos de mediación cuando se presentan parejas con historial de violencia. Este hecho contrasta con las posturas de algunos mediadores que plantean la imposibilidad de llevar a cabo procesos de mediación cuando existe o ha existido violencia; esto ha ocurrido debido a que la mirada predominante con respecto al tema de la violencia en el ámbito de la mediación familiar ha sido la perspectiva de género en la que la mujer tiene el rol víctima y el hombre el de victimario con una relación de sometimiento de uno hacía el otro; esta es una pauta de violencia complementaria donde la violencia sería utilizada como castigo. En este modelo existiría, en la base, un desequilibrio de poder que haría inviable la mediación, pues uno de los principios para realizar el proceso es que exista equilibrio de poder entre los participantes.

En relación a lo antes planteado, el haber identificado, por medio de este estudio, que la dinámica de violencia preponderante en las parejas que asistieron a mediación era la de violencia recíproca o cruzada y que la pauta imperante es simétrica con violencia

${ }^{21}$ Perrone, R. y Nannini, M., Violencia y abusos sexuales en la familia, Paidós, Buenos Aires, 2005, pp. 33-48.

22 Perrone, R. y Nannini, M., Violencia y abusos sexuales en la familia, Paidós, Buenos Aires, 2005, pp. 33-48. 
agresión lleva a replantear la perspectiva desde la cual se analiza la violencia de pareja en el ámbito de la mediación familiar, pues supone extinguir del imaginario los conceptos de desigualdad, víctima-victimario, culpabilidad y castigo, característicos de la mirada de género. Por el contrario, se hace necesario instalar nociones como pauta relacional, responsabilidad compartida, solución conjunta del problema, las cuales son propias de modelos teóricos integradores y sistémicos. Este cambio de paradigma supone considerar a las personas con capacidad para responsabilizarse de sus actos, devolviéndoles el poder para revertir conductas dañinas, lo que tendría impacto positivo para la reestructuración de pautas relacionales y el desarrollo de mecanismos de resolución de conflictos más eficaces y funcionales.

La información que arroja esta investigación constituye un aporte para el ámbito de la mediación familiar pues pone de relieve la necesidad de que los profesionales mediadores se capaciten y formen en la temática de violencia de pareja, con el objetivo de adquirir mayores destrezas y de esta forma realizar intervenciones que no sean iatrogénicas para los participantes y, lo que es más importante, estén preparados para no continuar invisibilizando un fenómeno que puede estar a la base de la relación. Es necesario señalar que el abordar casos de mediación familiar con antecedentes de violencia requiere de especialización y ciertas habilidades, entre las que están la aptitud para identificar y filtrar posibles casos de violencia donde exista desequilibrio de poder y se encuentre en riesgo la integridad física y/o psicológica de alguno de los participantes, además requiere del establecimiento de criterios para determinar en qué casos es viable la mediación y evaluar la capacidad de las partes para negociar efectivamente, evitando el riesgo de que la violencia se reanude.

Finalmente, y a modo de conclusión, el presente estudio se constituye en la primera investigación centrada en la temática de mediación familiar y violencia de pareja en nuestro país, haciendo un aporte en los ámbitos teóricos y prácticos pues permite generar conocimiento de las dinámicas de violencia presentes en las parejas que asisten a mediación familiar, estableciendo la necesidad de incorporar en la praxis profesional distintos análisis del fenómeno de violencia como base para el desarrollo de estrategias de intervención más eficaces.

\section{BIBLIOGRAFÍA}

Arredondo, V., Millán, R. y Lira, G., "Violencia Intrafamiliar: Un estudio exploratorio de factores sistémicos", en Anales V Congreso Iberoamericano de Psicologia Jurídica, Servicios Gráficos, Santiago de Chile, 2003, pp. 493-504.

Bernal, T., La mediación. Una solución a los conflictos de ruptura de pareja, Colex, Madrid, 1998, pp. $23-27$.

Bernal, T., "La mediación en ruptura de pareja", en Revista Centro de Resolución Alternativa de Conflictos, vol. 4, 2003, pp. 67-88.

Calquín. C., "Perfil psicosocial de la población usuaria del programa de atención y prevención de violencia intrafamiliar durante el año 2006 en Lo Espejo, Región Metropolitana de Santiago", Chile, en Revista Terapia Psicológica, 2007, vol. 25, pp. 85-93. 
CÁrdenas, E., La mediación en conflictos familiares, Lumen, Buenos Aires, 1998, pp. 209-235.

Cid, A., Momberg, M. y Salazar, D., "Mediación y VIF: de la negación a la intervención”, en Revista Centro de Resolución Alternativa de Conflictos, vol. 5, 2005, pp. 125-132.

Curi, S. y Gianella, C., "Mediación y violencia familiar en el contexto judicial", en Revista La Ley, vol. 3, 2002, pp. 305-315.

GonZÁlez-Capitel, C., Manual de Mediación, Atelier, Barcelona, 1999, pp. 17-29.

Haz, P., Violencia intrafamiliar, Presidencia de la República, Colombia, 2000, pp. 73-78.

Ortemberg, O., Mediación en la violencia familiar y en la crisis de la adolescencia, Editorial Universidad, Buenos Aires, 2002, pp. 59-82.

Perrone, R. y Nannini, M., Violencia y abusos sexuales en la familia, Paidós, Buenos Aires, 2005, pp. 33-48.

Pueyo, A.; López, S. y Álvarez, E., "Valoración del riesgo de violencia contra la pareja por medio de la SARA", en Papeles del Psicólogo, vol. 29, 2008, pp. 107-122.

Pueyo, A.; Redondo, S., "Predicción de la violencia: Entre la peligrosidad y la valoración del riesgo de violencia", en Papeles del Psicólogo, vol. 28, 2007, pp. 157-173.

Suares, M., Mediando en sistemas familiares, Paidós, Buenos Aires, 2002, pp. 373-387.

Vizcarra, M.; Cortés, J.; Bustos, L.; Alarcón, M. y Muñoz, S., "Violencia conyugal en la ciudad de Temuco. Un estudio de prevalencia y factores asociados", en Revista Médica de Chile, vol. 129 (12), 2001, pp. 1405-1412. 